\title{
VALLEy Forge STEEL: STRATEGy, RisK AND REWARD? A GRADUATE CASE IN STRATEGY AND MANAGEMENT ACCOUNTING
}

\author{
Patrick O’Meara
}

BRC Journal of Advances in Education 4, no. 1 (2020): 87-95. https://dx.doi.org/10.15239/j.brcacadjb.2020.04.01.ja06

WEB APPENDIX

https://dx.doi.org/10.15239/j.brcadvje.2020.04.01.wa06 
Table One ${ }^{i}$

\begin{tabular}{|l|l|}
\hline \multicolumn{2}{|c|}{ United States Government Treasury Yields (August, 2019) } \\
\hline Three-month Treasury & $1.999 \%$ \\
\hline Two-year Treasury & 1.634 \\
\hline Five-year Treasury & 1.527 \\
\hline Ten-year Treasury & $1.623 \%$ \\
\hline $\begin{array}{l}\text { Source: } \text { https:/www.cnbc.com/2019/08/14/the-inverted-yield-curve-explained-and-what- } \\
\text { it-means-for-your-money.html (August 18 2019) }\end{array}$ \\
\hline
\end{tabular}

Table Two

\begin{tabular}{|l|l|}
\hline \multicolumn{2}{|c|}{ Required New Production Staff } \\
\hline Shift & Number of New Production Staff \\
\hline Shift One (7-3p.m.) & 20 \\
\hline Shift Two (3-11p.m.) & 20 \\
\hline Shift Three (11p.m.-7a.m.) & 12 \\
\hline Total & $\mathbf{5 2}$ \\
\hline Assuming a 40-hour work week over 48 weeks per year \\
\hline
\end{tabular}

Table Three

\begin{tabular}{|l|l|}
\hline \multicolumn{2}{|c|}{ Current Management and Non-Production Staff Salaries } \\
\hline Manager Shift One & $\$ 105,000$ \\
\hline Manager Shift Two & 95,000 \\
\hline Night Shift Supervisor & 77,000 \\
\hline Plant Accountant & 90,000 \\
\hline Bookkeeper & 38,000 \\
\hline $\begin{array}{l}\text { Manager and Plant Accountant Benefits: } \mathbf{2 0} \% \text { of Salary } \\
\text { Bookkeeper: 15\% of Salary }\end{array}$ \\
\hline
\end{tabular}


Table Four

\begin{tabular}{|l|l|}
\hline \multicolumn{2}{|c|}{ Current Contract Staff Costs } \\
\hline Plant Security Staff & $\$ 80,000$ \\
\hline Cleaning Contract & 30,000 \\
\hline Commissioned Sales Staff & $5.00 \%$ of sales \\
\hline No benefits for contract staff \\
\hline
\end{tabular}


Table Five $e^{i i}$

\begin{tabular}{|l|l|}
\hline \multicolumn{2}{|c|}{ Cost of Direct Materials } \\
\hline Coking Coal (per m.t) & \$191 USD \\
\hline Iron Ore (per m.t) & \$117.50 USD \\
\hline Limestone (per one-half m.t.) & \$13 USD \\
\hline $\begin{array}{l}\text { Source: } \text { https://www.focus-economics.com/commodities/energy/coking-coal (August, } \\
\text { 2019) }\end{array}$ \\
\hline
\end{tabular}

Table Six ${ }^{i i i}$

\begin{tabular}{|l|l|}
\hline \multicolumn{2}{|c|}{ Historical Market Price for Aluminum (USD p.m.t) } \\
\hline $\mathbf{2 0 1 3}$ & $\$ 1845.86$ \\
\hline $\mathbf{2 0 1 4}$ & $1,867.48$ \\
\hline $\mathbf{2 0 1 5}$ & $1,663.69$ \\
\hline $\mathbf{2 0 1 6}$ & $1,604.60$ \\
\hline $\mathbf{2 0 1 7}$ & $1,968.40$ \\
\hline $\mathbf{2 0 1 8}$ & $2,107.64$ \\
\hline August, 2019 & $1,784.00$ \\
\hline $\begin{array}{l}\text { Source: https:/Www.focus-economics.com/commodities/base-metals/aluminium (August, } \\
\mathbf{2 0 1 9 )}\end{array}$ \\
\hline
\end{tabular}

Table Seven ${ }^{\text {iv }}$

\begin{tabular}{|l|l|}
\hline \multicolumn{2}{|c|}{ Historical Market Price for Steel (USD per m.t) } \\
\hline $\mathbf{2 0 1 3}$ & $\$ 630.41$ \\
\hline $\mathbf{2 0 1 4}$ & 653.14 \\
\hline $\mathbf{2 0 1 5}$ & 461.14 \\
\hline $\mathbf{2 0 1 6}$ & 519.95 \\
\hline $\mathbf{2 0 1 7}$ & 619.92 \\
\hline $\mathbf{2 0 1 8}$ & 828.46 \\
\hline August, 2019 & 525.00 \\
\hline $\begin{array}{l}\text { Source: https:/Www.focus-economics.com/commodities/base-metals/aluminium (August, } \\
\mathbf{2 0 1 9 )}\end{array}$ \\
\hline
\end{tabular}

Table Eight ${ }^{v}$

\begin{tabular}{|l|l|l|}
\hline \multicolumn{3}{|c|}{ Sales Growth (Decline) By Recession Scenario for Valley Forge } \\
\hline & Sales Growth (Decline) & Probability \\
\hline Mild Recession & $0 \%$ sales growth & $30 \%$ \\
\hline Moderate Recession & $1 \%$ sales growth & $30 \%$ \\
\hline Strong Recession & $3 \%$ sales decline & $40 \%$ \\
\hline $\begin{array}{l}\text { Note: Between 1945 and 2010, the average length of a recession in the United States has } \\
\text { been 18 months. } \\
\text { Source: https://www.nber.org/cycles.html }\end{array}$
\end{tabular}




\section{Endnotes}

i https://www.cnbc.com/2019/08/14/the-inverted-yield-curve-explained-and-what-it-means-foryour-money.html (August 18 2019)

ii https://www.focus-economics.com/commodities/energy/coking-coal (August, 2019) iii https://www.focus-economics.com/commodities/base-metals/aluminium (August, 2019) iv https://www.focus-economics.com/commodities/base-metals/aluminium (August, 2019) v https://www.nber.org/cycles.html 